\title{
Toward long-term laptop performances
}

Khaled Moustafa

Editor of ArabiXiv (https://arabixiv.org)

Email: Khaled.moustafa@arabixiv.org

\begin{abstract}
To improve laptops' performances over time and to keep them continuously upgradable, I suggest a new laptop design that includes external slots dedicated for CPU (processor), RAM (memory), storage and graphics cards that should be removable and connectable to their internal counterparts to enhance their functionalities whenever needed. With each new generation of these components, laptops can therefore be easily upgradable and improvable to be up to date in terms of functions, performance and versatility.
\end{abstract}

Keywords: computer architecture; laptop optimization; long-term laptop evolution;

Handheld smartphones have changed users' behaviors on how, where and when to communicate with each other. With different kinds of applications (Apps) in the various fields, mobile devices are getting more powerful every day. However, smartphones can't fully replace traditional desktops or laptops for professional uses in terms of performance, flexibility and versatility. Laptops or personal computers (PCs) are nowadays one of the essential devices in professional work environments and entertainment lifestyle.

To adapt with everlasting changes in hardware and software industry, I suggest a new laptops design/architecture with a permanent ability to improve/upgrade PC's performances relating to the main following characteristics through external/lateral slots as shown in figure 1 :

- An external slot for RAM (Random-Access Memory) to upgrade/enhance the internal RAM capabilities.

- An external slot for the CPU (central processing unit=processor) to upgrade/increase the internal processor speed and performance. 
- An external slot for storage drive (SD) to increase the internal storage capacity. Some laptops already offer this possibility.

- An external slot for Graphics card to enhance/upgrade the internal graphics and 3D performances.

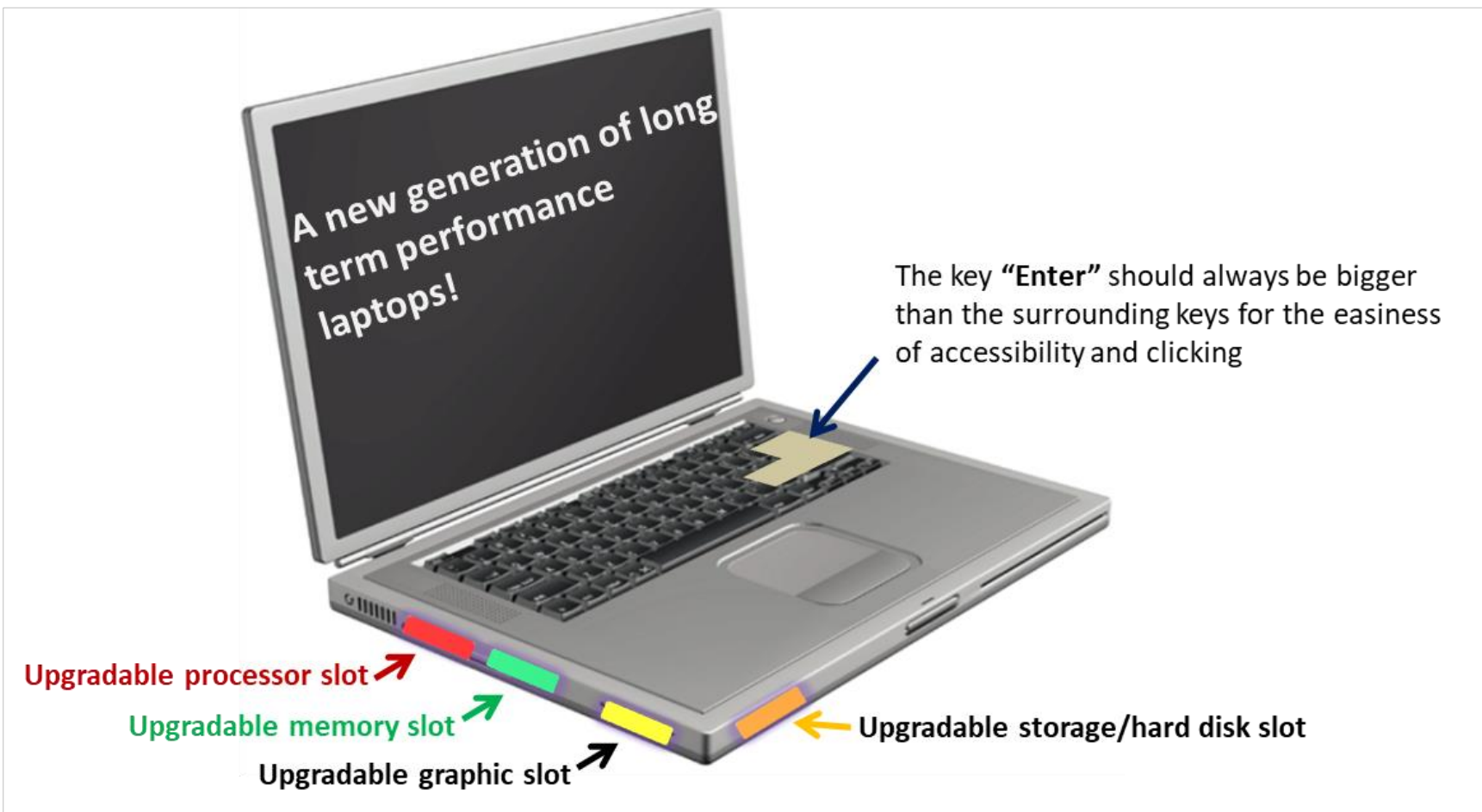

Figure 1. A proposal to enhance laptops' performances with external dedicated slots for removable/upgradable processor, random memory, graphics and storage drives.

The speed, memory, graphics and storage are important characteristics in desktop and laptop computers. To enhance laptop evolvability and performances, laptops can be manufactured with external slots dedicated to external/replaceable processor stick, memory "RAM" stick, graphics card, and storage disk. So, when new generations of these components are available, users can upgrade them in an easy and simple way. Miniaturization and standardization of these components in SD-format size might be a good option to go with. The emplacements can be on the side, the front or the back of the laptop's chassis as shown in the figure above. The emplacements can be distributed on the four sides of the laptop in coherence with other slots for USB, Ethernet Port, etc.

Manufacturing laptops with the possibility to upgrade these components will make laptops highly performant and environment-friendly devices to reduce computer industry carbon footprint. With such improvements, laptops' constructors and users will need to change/upgrade a few components only -the four aforementioned components- rather than to replace the whole machine, reducing the environmental carbon impact caused by this industry. Long-term improvable laptops' performances will also allow any new resource-intensive applications and large files such as large databases, bioinformatics' tools and applications that consume high 
CPU resources, videos editing and 3D applications to install and run anytime without much hurdles.

Some laptops already offer the possibility to upgrade internal hard disks and/or memory sticks, with some easiness/difficulties. However, the majority of laptops, particularly thin ones, do not offer such a possibility or only experienced users can do it, if any, which reduces laptops' polyvalence and performance after a few years of their manufacturing. Other laptops' manufacturers make it almost impossible to upgrade any laptop's components by soldering them on the motherboard. This restriction renders laptop's live cycles relatively short with important environmental considerations behind.

Therefore, to fulfill the requirements of speed, storage, memory, and graphics features that change over time, laptops should be resilient, flexible and upgradable continuously. For this, laptops need to be manufactured with the idea that they should be easily cleanable, easily upgradable and easily repairable, starting from the keyboard's buttons to the processor. Lateral slots dedicated to external and always replaceable/upgradable processor, memory, storage and graphics chipsets to connect to, and expend, the built-in characteristics would be a major step forward in sustainable laptops' performances and industry.

On another hand, laptops constructors need to rethink the way the buttons of the keyboards are currently manufactured and fixed on the laptops' chassis/motherboard. In all laptop brands, it is particularly difficult to remove the keys and to clean the space between them or under them. A reasonably large space between the keys is generally comfortable for typing, but over time it may accumulate dust particles that need to be removed regularly between and under the keys. However, removing the keys and reinserting them is currently a painful process and time consuming. For a long-term maintenance, laptops manufacturers should thus make it easy to remove the keyboard buttons and to reinsert them painlessly.

\section{Disclaimer: None}

